$536.22: 532.542$

\title{
Heat Transfer in an Annulus with an Inner Rotating Cylinder*
}

\author{
By Fujio Tachibana**, Sukeo Fukui***, \\ and Hisao MrTSUMURA***
}

The heat transfer by the convection in the annular gap between the rotating inner cylinder and the stationary outer cylinder was measured in the air, spindle oil and mobile oil at varying rotational speeds, cylinder diameters and widths of gap. The results show that characteristics of the flow and heat transfer have two modes.

If the rotational speed is low and the width of gap is narrow, namely $(v \delta / \nu) \sqrt{\delta / r}<41$, the flow is laminar and the heat is transferred by conduction and radiation.

But if $(v \delta / \nu) \sqrt{\delta / r}>41$, the rate of heat transfer increases with rotational speeds under the influence of secondary vortices induced by the centrifugal force, which may be given by

$$
\frac{U \delta}{\lambda}=0.21\left(\frac{v^{2} \delta^{2}}{\nu^{2}} \cdot \frac{\delta}{r} \cdot P_{r}\right)^{1 / 4}
$$

where $U$ denotes the overall coefficient of heat transfer through the gap, $\delta$ the width of the gap, $\lambda$ the thermal conductivity, $\nu$ the kinematic viscosity and $P_{r}$ the Prandt1 Number, $r$ and $v$ the radius and the peripheral velocity of the rotating cylinder respectively.

\section{Nomenclature}

The following nomenclature is used in the paper:

$A:$ cylindrical surface area of the test section, $\mathrm{m}^{2}$

$F:$ view factor

$g:$ acceleration due to gravity $\mathrm{m} / \mathrm{sec}^{2}$

$G_{r}$ : Grashof Number $\dagger g \beta \Delta t \partial^{3} / \nu^{2}$

$N_{u}:$ Nusselt number, $U \hat{\partial} / \lambda$

$P_{r}:$ Prandtl number

$Q:$ overall heat transfer rate, $\mathrm{kca} 1 / \mathrm{hr}$

$Q_{e}$ : heat transfer rate by convection, $\mathrm{kcal} / \mathrm{hr}$

$Q_{r}$ : heat transfer rate by radiation, $\mathrm{kcal} / \mathrm{hr}$

$r:$ radius of the inner cylinder, $m$

$R_{\theta}:$ Reynolds number, $v \delta / \nu$

$\Delta t:$ temperature difference between the inner and the outer cylinder, ${ }^{\circ} \mathrm{C}$

$T_{1}, T_{2}$ : temperature of the inner and the outer cylinder, ${ }^{\circ} \mathrm{K}$

$T_{a}:$ Taylor number $(v \delta / \nu) \sqrt{\delta / r}$

* Received 15 th April, 1959

** Professor, The Institute of Industrial Science, University of Tokyo.

*** Mechanical Engineer, Tsurumi Research Laboratory, Tokyo Shibaura Electric Co., Ltd., Tsurumiku, Yokohama.

$\dagger$ Physical properties of fluid are taken at the mean temperature of both cylinder suriaces.
$U$ : overall coefficient of heat transfer, $Q_{c} / A \cdot \Delta t, \mathrm{kcal} / \mathrm{m}^{2} \mathrm{hr}{ }^{\circ} \mathrm{C}$

$v:$ peripheral velocity of the inner rotating surface, $\mathrm{m} / \mathrm{sec}$

$\beta$ : coefficient of volumetric expansion, $1 /{ }^{\circ} \mathrm{C}$

$\delta:$ width of the annular gap, $\mathrm{m}$

$\varepsilon:$ emissivity,

$\lambda:$ thermal conductivity of fluid, $\mathrm{kcal} / \mathrm{m} \mathrm{hr}{ }^{\circ} \mathrm{C}$ $\mu:$ absolute viscosity, $\mathrm{kg} / \mathrm{m} \cdot \mathrm{sec}$

$\nu:$ kinematic viscosity, $\mathrm{m} / \mathrm{sec}^{2}$

\section{Introduction}

The heat transfer by convection in the annular gap between the rotating inner cylinder and the stationary outer cylinder occurs in various rotating machines, such as electric motors. For the design of electric machines the analysis of this heat transfer is one of the most important problems in order to reduce the size and increase the efficiency. Although there are a few experimental data for the heat transfer in such an annulus, the results are contradictory.

In 1923 G. I. Taylor ${ }^{(5)}$ studied theoretically the stability of the gap flow and concluded that when the Reynolds number exceeds the critical value for a given ratio of the width of the gap to the inner cylinder, vortices were generated in the 
annulus. The critical value is expressed by

$\left(R_{e}\right)_{\mathrm{cri}}=41.1(r / \delta)$

J. Kaye and E. C. Elgar ${ }^{(3)}$ have reported about the flow of annular gap with a rotating inner cylinder by the hot wire method and photographs, confirming the Taylor's prediction. Fig. 1 is the schematic picture of vortices which are generated in the gap. The heat transfer characteristic was studied; however, the results were presented qualitatively but not quantitatively. C. Gazley ${ }^{(1)}$ has obtained experimentally the coefficient of the friction and that of the heat transfer when the axial flow was superposed on the flow of the inner rotating cylinder which had the slotted surface resembling the rotor of the electric motor in addition to the smooth surface. When the inner and outer surfaces are smooth and the width of the gap is $6.1 \mathrm{~mm}$ and $0.43 \mathrm{~mm}$, the results of experiments are arranged by the correlation of Nusselt Number and Reynolds Number; however, its correlation is not so consistent for the cases of different gaps.

The present investigation has the objective to correlate, as a simplified case, the heat transfer between the inner rotating cylinder and the stationary outer cylinder without the axial flow with the various gap, rotational speed, radius of the cylinder and kind of fluid.

\section{Experimental equipment}

The test equipment consists of the inner rotating cylinder and the outer stationary cylinder coaxial with it. The rotating cylinder is heated by the inner electric heater and the stationary one has the water jacket for dissipating the heat transmitted. Fig. 2 presents the outline of the test equipment. The test equipments are two. One has the rotor of $58 \mathrm{~mm}$ diameter and the other has $120 \mathrm{~mm}$ diameter. But both constructions are the same except the location of the brush and some of the fitting methods.

The test cylinder is $210 \mathrm{~mm}$ long and $120 \mathrm{~mm}$ in diameter and has a smooth and black colored surface. The nichrome wire wound on the bobbin is enclosed in its center with asbestos. The compensation cylinders are connected with both sides

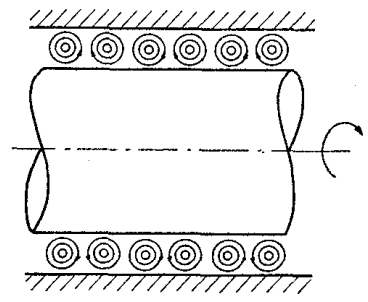

Fig. 1 Gap flow of the test cylinder through insertion of a bakelite plate of $5 \mathrm{~mm}$ thick to prevent the axial heat flow. They are $120 \mathrm{~mm}$ in diameter and $83 \mathrm{~mm}$ long, and the heaters in them are enclosed with asbestos.

Outer stationary cylinder is concentric and of the same length with the inner one including the compensation cylinders. Its inner diameters are four: $124,144,160$ and $230 \mathrm{~mm}$; and its surface is smooth and red colored and it is cooled from outside by water. The rotationary cylinder is held horizontally by bearings at both ends and the slip rings are outside of them, being driven by the pulley at one end.

The heater in the rotational one is heated electrically. The lead of the nichrome wire is connected to the slip ring through the inner cylinder. The slip ring is made of brass and the brush is of silver-graphite. The three heaters have each a slider resistor, and are heated by alternating current. The input of electricity to the test cylinder is calculated by the current and the voltage drop of the tap. The input to the compensation cylinders is controlled manually when the temperature difference between the test and the compensation cylinders arises. The meter of current and voltage is read with accuracy to $\pm 1 \%$.

The surface temperature of the rotationary cylinder is obtained by the variance of the resistance of the thermistor which is buried in its surface. The temperature difference between the test cylinder and the compensation cylinder is measured by the thermocouples soldered on the surface. Its value is obtained accurately to 0.01 millivolts. The resistance of the thermistor is compared with the temperature which is measured by the accurate thermocouple buried in the surface opposite the location of the thermistor. As its value is $10 \sim 80$. $\mathrm{k} \Omega$ usually at the room temperature, the contact resistance that considerably affects the accuracy of the thermocouple method offers no problem.

The temperature of the outer cylinder surface is equal to the mean temperature of inlet and outlet water, and its difference does not exceed

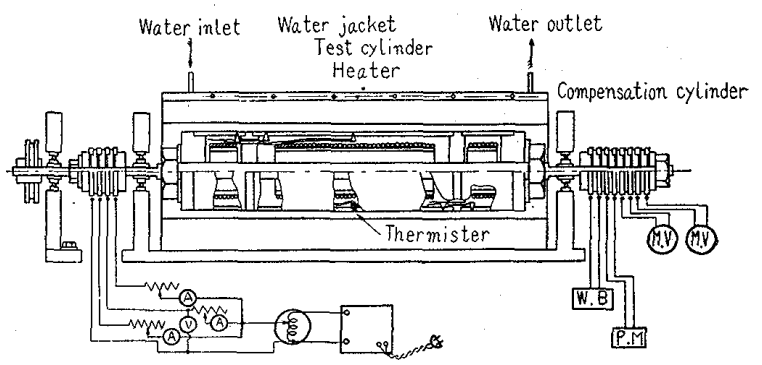

Fig. 2 Details of construction of test equipment 
one degree centigrate. The temperature difference between inner and outer ones was about $20^{\circ} \mathrm{C}$ or $30^{\circ} \mathrm{C}$ in our experiments. These experiments were mostly conducted in the air. When the spindle oil and mobile oil are used, the test equipments are immersed in the oil, therefore the oil film grows on the surface of slip rings and the needle of the meter oscillates when the rotation reaches about 1000 r.p.m., making the measurement very hard.

The widths of the annular gap are five: $0.88,1.97,4,6$, and $10 \mathrm{~mm}$ in the cylinder of $58 \mathrm{~mm}$ diameter and four; $2,12,20$, and $55 \mathrm{~mm}$, in the one of $120 \mathrm{~mm}$ diameter.

\section{Experimental results}

When the inner rotating cylinder is heated and the outer stationary one is cooled from outside without the axial flow, the heat transfer of the annular gap between the inner and the outer cylinder is correlated and our experimental equation is found applicable for the air, spindle oil and mobile oil.

When the air is used, the heat transferred by radiation must be corrected by calculating, so it becomes

$$
Q_{r}=\varepsilon \cdot F 4.88\left[\left(\frac{T_{1}}{100}\right)^{4}-\left(\frac{T_{2}}{100}\right)^{4}\right] A
$$

Then the heat by convection $Q_{c}$ is

$$
Q_{c}=Q-Q_{r}
$$

The heat transfer in the air, spindle oil and mobile oil was evaluated for a range of Reynolds number from 0.84 to $4.7 \times 10^{4}$ and Taylor number from 0.49 to $4.5 \times 10^{4}$. Then the rotational speed is from 3 to 2840 r.p.m., and the radius of the inner cylinder is $29 \mathrm{~mm}$ and $60 \mathrm{~mm}$.

When the rotational speed is low and the gap is narrow in the air, the overall heat transfer coefficient is smaller. Radiation and conduction occupy the most part of the whole heat transfer. Accordingly the heat transfer is not affected by the rotational speed.

When the rotational speed increases and the width of the gap becomes larger and then the square of the Taylor number exceeds about 1700 , the secondary vortex is generated by the centrifugal force and the coefficient of the heat transfer increases with velocity increase. Here the Taylor

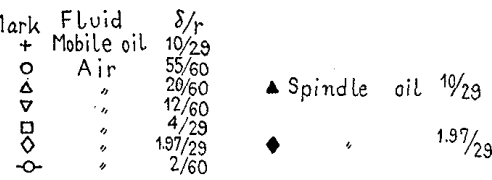

$97 / 29$

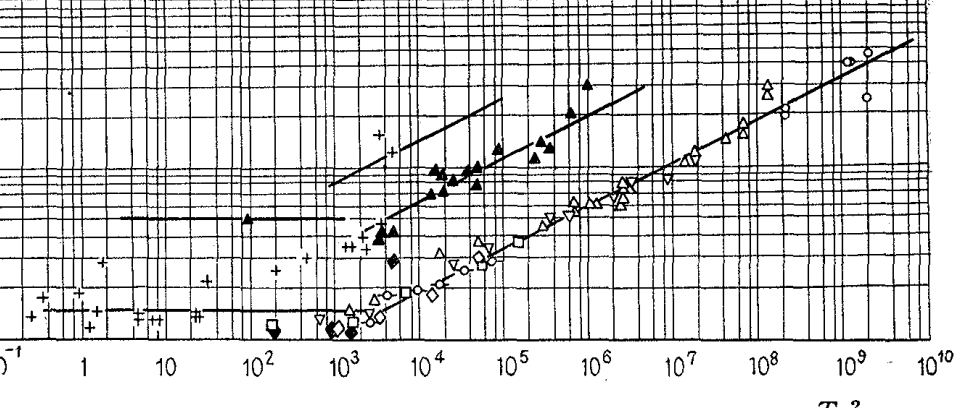

Fig. 3 Test results for heat transfer

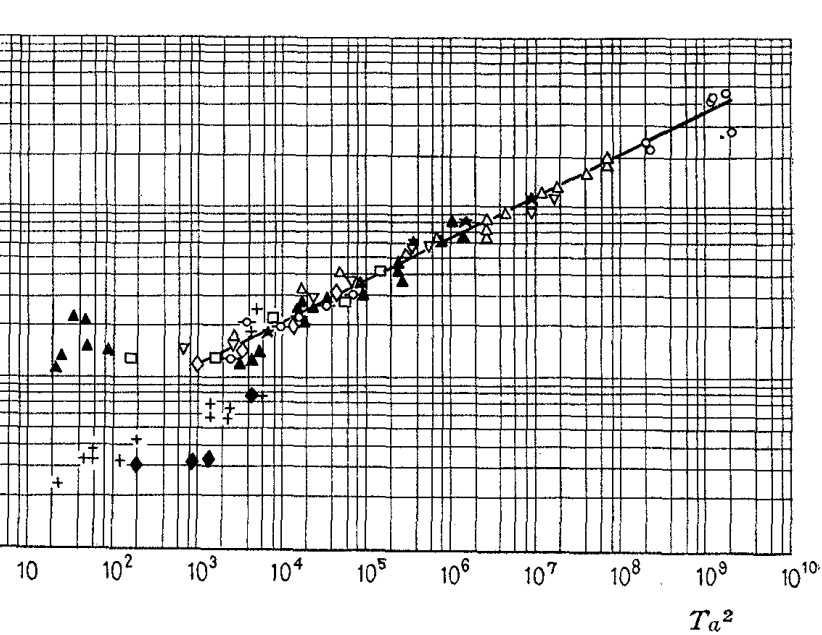

Fig. 4. Correlation of the test results

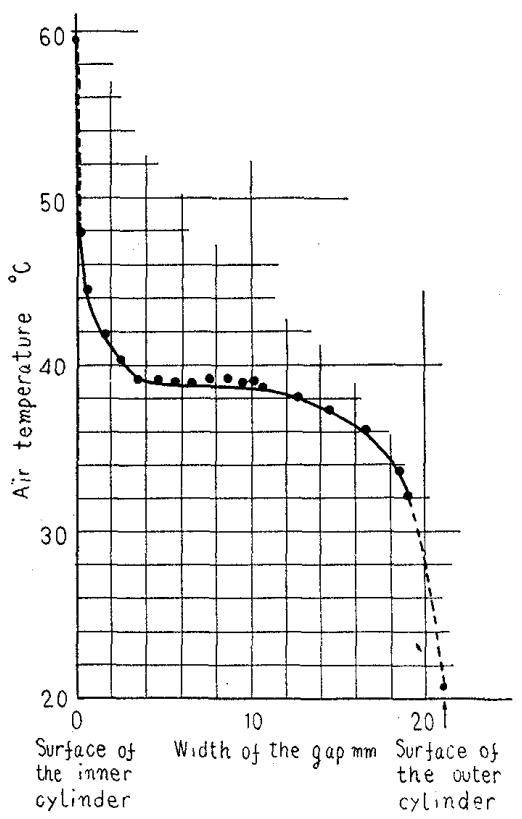

Fig. 5 Temperature distribution in an annular gap, with inner rotating cylinder 
number is a dimensionless group defined by $(v \delta / \nu) \sqrt{\hat{\partial} / r}$. In this region radiation and conduction become less, and the convection of the secondary vortex is predominating. It is the same for the spindle oil and mobile oil. As shown in Fig. 3 the experimental data are correlated by the Nusselt number and the Taylor number.

By this correlation the influences of the radius, the width of the gap and the rotational speed are represented by one straight line. However, because the inconsistency of Nusselt number among the various fluids remains, the data are crossplotted and rearranged by the Prandtl number of fluid and represented in Fig. 4. In this Figure the ordinate is $N_{u} / P_{r}^{1 / 4}$ and the abscissa is $T_{a}^{2}$. For $T_{a}^{2}>1700$, the data give good agreement with the equation:

$$
N_{u}=0.21\left(T_{a}^{2} \cdot P_{r}\right)^{1 / 4}
$$

This flow in the annulus is a secondary steady flow in the form of pairs of counter-rotating vortices and its vortices continue regularly in the stripe-pattern. And the temperature distribution in the annular gap is shown in Fig. 5 . In this Figure the radius is $60 \mathrm{~mm}$, the width of the gap $20 \mathrm{~mm}$, and the rotational speed 460 r.p.m. A considerable temperature change occurs at the 1 or $2 \mathrm{~mm}$ distance from the surface and the other part of the center is almost flat. This shows the mixing by the vortices.

This mechanism of the heat transfer in the annular gap is similar to that of the air gap of the enclosed horizontal parallel planes when the heat is transferred from the lower one to the upper one. For this case the next equation is given by Jakob(2).

$$
\left.\begin{array}{l}
U \partial / \lambda=0.195 G_{r}{ }^{1 / 4} \\
4 \times 10^{5}>G_{r}>10^{4}
\end{array}\right\}
$$

where $U$ is the overall heat transfer coefficient from lower surface to upper one. As the motive force of convection is the centrifugal force in the case of a rotating cylinder, the centrifugal force term, $v^{2} / r$, is substituted instead of the term of the buoyancy force $g \beta \Delta t$ in the Eq. (4). Then:

$$
\frac{U \delta}{\lambda}=0.195\left(\frac{v^{2} \partial^{2}}{\nu^{2}} \cdot \frac{\partial}{r}\right)^{1 / 4}
$$

This equation is applicable only for the air, so it is rewritten by assuming that $P_{r}$ is 0.71 , as follows:

$$
\frac{U \delta}{\lambda}=0.211\left(\frac{v^{2} \delta^{2}}{\nu^{2}} \cdot \frac{\delta}{r} \cdot P_{r}\right)^{1 / 4}
$$

This is quite the same as the Eq. (3). Furthermore this equation can be applied up to $10^{10}$ of the square of Taylor number even though exceeding the Grashof number limit $4 \times 10^{5}$.

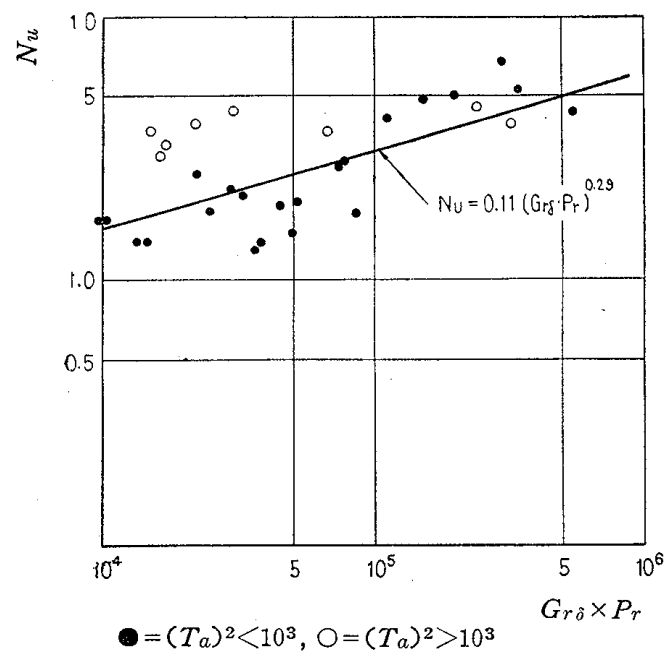

Fig. 6 Natural convection from horizontal annulus with inner rotating cylinder

When the rotational speed is low and the width of the gap is narrow, accordingly $T_{a}^{2}<1700$, the flow of the annulus is laminar the heat transferred by radiation and conduction is predominating and in addition the effect of the natural convection is superposed. For the air this effect is so small, but for the spindle and mobile oil it is rather great. As the Grashof number multiplied by the Prandtl number exceeds $10^{4}$, our experimental data agree well with the equation of H. Kraussold ${ }^{(4)}$ :

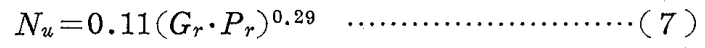

They are shown in Fig. 6. But when $T_{a}^{2}>1000$, the heat transfer coefficient is too high to correlate this equation as the flow of the natural convection and the rotationary one are superposed on each other.

The data by Gazley $^{(1)}$ which were obtained from an experiment for the air with the width of the gap, $6.1 \mathrm{~mm}$ and $0.43 \mathrm{~mm}$, are recalculated and shown in Fig. 4, presenting a good agreement with our experimental Eq. (3).

\section{Conclusions}

The heat transfer by the convection in the annular gap between the rotating inner cylinder and the stationary outer cylinder was experimented for the air, spindle oil, and mobile oil at various rotational speeds, cylinder diameters and widths of gap. By the experiment we obtained the results mentioned below:

1) The characteristics of this flow and heat transfer have two regions:

(1) Laminar region, in which the flow is laminar and the heat transferred by radiation and conduction predominates.

(2) Vortex region, in which the flow has 
counterrotating vortices in regular rows and the heat transfer is controlled by such convection as increases with the rotational speed. The transition from laminar to vortex region is decided only by the Taylor number. The critical Taylor number is 41 .

2) When $T_{a}<41$, the heat transfer by the natural convection due to the temperature difference of both walls is superposed accompanying the increase of the Rayleigh number $\left(G_{r} \cdot P_{r}\right)$, and for the oil this effect is fairly great. When $\left(G_{r} \cdot P_{r}\right)>10^{4}$, the data are correlated by the following equation:

$$
N_{u}=0.11\left(G_{r} \cdot P_{r}\right)^{0.29}
$$

But for the air the Rayleigh number is small, therefore the natural convection offers no problem.

3) When $T_{a}>41$, the overall heat transfer coefficients is correlated by the experimental equation,

$$
N_{u}=0.21\left(T_{a}^{2} \cdot P_{r}\right)^{1 / 4}
$$

\section{References}

(1) C. Gazley: Trans. ASME, Vol. 80 (1958), p. 79.

(2) M. Jakob: Heat Transfer I, (1949) p. 535.

(3) J. Kaye and E.C. Elgar: Trans. ASME, Vol. 80 (1958), p. 753.

(4) H. Kraussold: Forschung, Bd. 5, Ht. 4 (1934), p. 186.

(5) G.I. Taylor: Phil. Trans. Roy. Soc., series A, Vol. 223, (1923), p. 289

621. 436.038

\title{
Studies on the Penetration of Fuel Spray in a Diesel Engine*
}

\author{
By Yutaro Wakuri**, Masaru FujiI**, Tatsuo Amitani***, \\ and Reijiro TSUNEYA***
}

\begin{abstract}
Regarding the penetrating distance of fuel spray in a diesel engine, the old theory dealing with the motion of a fuel droplet in still air is recognized not to coincide with the actual phenomenon in a diesel engine because of the extremely small size of atomized fuel droplets and the very high density of gas in cylinder. In this paper, the characteristics of spray penetration are discussed from the viewpoint of momentum theory based on the idea that the air induced into a fuel jet stream makes a kind of mixed gas together with fuel droplets. According to the results of experiment, the authors confirmed that the theory was satisfactory, that there existed the simple relations among several dimensionless numbers which indicate the effect of various factors on the spray penetration, that there is a close relationship between the spray cone angle and penetration, and others.
\end{abstract}

\section{Introduction}

Regarding the penetration of fuel spray in a diesel engine, various studies have been made by such investigators as Sass ${ }^{(1)}$, Miller \& Beardsley ${ }^{(2)}$, Schweitzer ${ }^{(3)}$ etc. The theoretical aspect of the conventional studies has been based on the idea that fuel spray moves in still air. However, in the case of such minute oil droplets as of diesel engine fuel spray with the initial diameter of 10

* Received 8 th April, 1959.

** Research Fellow, Laboratory, Mitsubishi Ship. building \& Engineering Co. Ltd., Nagasaki.

*** Designer, Designing Shop, Mitsubishi Shipbuilding \& Engineering Co. Ltd., Nagasaki Works. to 20 microns, if the theories were satisfactory, the velocity of the oil droplets injected in the combustion chamber should decrease suddenly and the droplet should not penetrate. However, actual experimental results do not show such sudden decrease in velocity. Therefore, taking up the momentum theory in which the mass of air is taken into account, the authors have examined the characteristics of the spray penetration.

\section{Momentum theory}

The resistance of the oil droplets $R$ is given by the following Eq. (1).

$$
R=\frac{\rho_{a}}{2} C_{D} \frac{\pi}{4} D^{2} V^{2}
$$

